\title{
Study of a thermo-acoustic instability triggering in a low-swirl burner using simultaneous time-resolved acetone and OH-PLIF.
}

\author{
Antoine Renaud ${ }^{\mathrm{a}, *}$, Takeshi Yokomori ${ }^{\mathrm{b}}$, Shigeru Tachibana ${ }^{\mathrm{c}}$ \\ ${ }^{a}$ Laboratoire EM2C, CNRS, CentraleSupelec \\ ${ }^{b}$ Faculty of Science and Technology, Keio University \\ ${ }^{c}$ Aeronautical Technology Directorate, Japan Aerospace Exploration Agency (JAXA)
}

\begin{abstract}
A low-swirl burner operating in premixed mode with methane and air is studied using high-speed acetone and $\mathrm{OH}-$ PLIF and advanced post-processing methods. In a transient operating point where the equivalence ratio is slowly increased while keeping the bulk velocity constant, a thermo-acoustic instability is found to grow and generate large pressure fluctuations in the combustion chamber. The instability is maintained by bursts of flame in phase with the pressure fluctuation cycle. It is found that these bursts are amplified by the fact that the boundaries of the flame arms are fluctuating in phase. This phasing of the arms' boundaries is due to the change in flame speed coming from the increase of equivalence ratio.
\end{abstract}

Keywords:

low swirl, time-resolved, DMD, Hilbert transform

\section{Introduction}

Lean premixed combustion is a widely acknowledged way to control nitrogen oxides emissions because it allows to control the combustion temperature and thus the production of thermal-NOx [1]. A main drawback of lean premixed flames is however their sensitivity to external perturbations and their propensity to trigger thermo-acoustic instabilities when used in confined conditions such as within the combustion chamber of a gasturbine. Thermo-acoustic instabilities can generate high pressure fluctuation levels leading to the flame blow-off or damages to the burner [2].

In the present study, a low-swirl burner is used. This kind of injectors, first developed at the Lawrence Berkeley National Laboratory [3, 4], is known for its reduced pollutant emissions [5] and may therefore prove interesting for gas turbine applications. Low swirl injectors also present promising properties regarding fuel flexibility as they can easily accomodate several types of

*Corresponding author: Antoine RENAUD; Laboratoire EM2C, CNRS, CentraleSupelec; 3 rue Joliot Curie; 91190 Gif-sur-Yvette, France

Email address: antoine.renaud@centralesupelec.fr (Antoine Renaud)

Preprint submitted to Proceedings of the Combustion Institute gaseous fuels such as methane, hydrogen or syngas [68]. From a more fundamental point of view, low-swirl injectors create a divergent flow without any inner recirculation zone, thus allowing to stabilize a freely propagating planar turbulent flame around the center of the burner and have therefore been used in detailed turbulent combustion studies [9].

The dynamical behavior of confined low-swirl flames has been the subject of several experimental and numerical studies [10-16]. Therkelsen et al. [14] analyzed $\mathrm{CH}_{4}$-air and $\mathrm{H}_{2} / \mathrm{CH}_{4}$-air flames that presented self-sustained thermo-acoustic instabilities. Thanks to phase-locked PIV measurements, they highlighted ring vortices periodically detaching from the burner rim in the external shear layer as the main mechanism leading to the instability.

Laser diagnostics are a key tool in combustion studies because of their minimal intrusiveness [17]. Highspeed Planar Laser-Induced Fluorescence (PLIF) gained momentum with the advances in high-speed dye lasers, image intensifiers and cameras and is now mature. This technique provides numerous advantages for the study of flame dynamics [18] and enables the use of well-established signal processing tools such as Fourier transform and its derivatives.

In the present study, as the equivalence ratio of 
a methane-air low-swirl flame in confined conditions is increased, the pressure fluctuations also grow because of the triggering of a thermo-acoustic instability. Using time-resolved laser-induced fluorescence diagnostics and advanced post-processing techniques, the growth of the instability is studied and is found to be linked to the increase in flame speed due to the increase of the equivalence ratio.

\section{Experimental Methods}

\subsection{Experimental Setup}

The setup used in this study is shown in Fig.1.

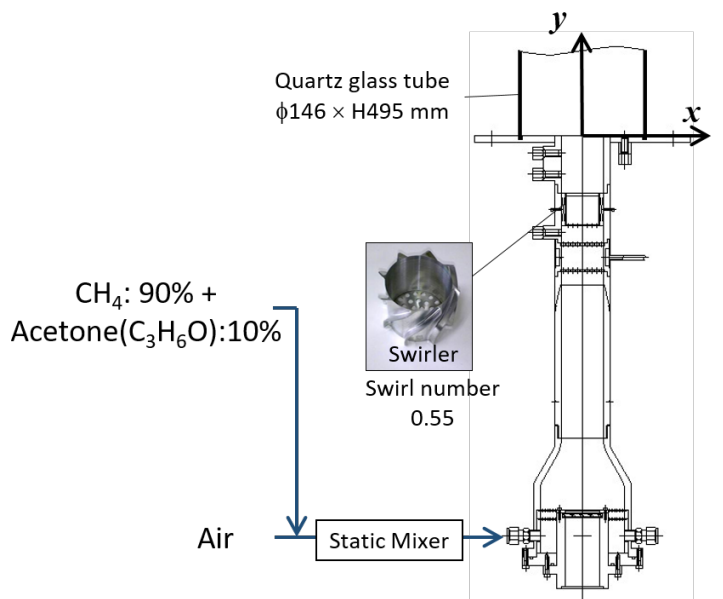

Figure 1: Schematics of the low-swirl burner.

The low-swirl burner uses a premixture of methane and air which is sent through a particular swirler, shown in a smaller picture in Fig.1. The outer part of the swirler has a swirl number of 0.55 to give the outer flow a rotational motion while the inner part has holes to let the mixture through. By effectively combining an axial injection and a swirl, this creates a divergent flow field in the combustion chamber without any recirculation zone. A turbulent flame can thus be stabilized aerodynamically. The exit diameter of the injector is 50 $\mathrm{mm}$ and the mixture flows in a $495 \mathrm{~mm}$-long cylindrical combustion chamber (diameter: $146 \mathrm{~mm}$ ) made of fused silica.

Because the behavior of the fresh gases is studied in the present work, $10 \%$ (in volume) of acetone is added to the fuel as a fluorescent tracer. This ratio is chosen to optimize the fluorescence signal without disturbing too much the combustion reaction [19].

To study the growth of a thermo-acoustic instability, a transient operating point is used. The bulk velocity at the exit of the injector is kept constant at $10 \mathrm{~m} \cdot \mathrm{s}^{-1}$. During the 4.4 second transient operation time, the equivalence ratio is progressively increased from 0.66 to 0.70. This results in a global power increase from 45 to $48 \mathrm{~kW}$.

\subsection{Diagnostics}

To allow for the simultaneous imaging of both fresh and burnt gases, a dual high-speed acetone and $\mathrm{OH}$ PLIF setup is used. Since acetone is added to the fresh mixture and burns at the flame front, a fluorescence signal from acetone means that fresh gases are present. On the contrary, $\mathrm{OH}$ is produced at the flame front and remains in the post-flame zone where it will be slowly oxidized, meaning that the $\mathrm{OH}$ fluorescence signal represents the reaction products. To excite both species, a $283.6 \mathrm{~nm}$ wavelength generated by a dye laser operated on Rhodamine (Sirah Credo-DYE-Amp-SH-FE) is used. The dye laser is pumped by a $10 \mathrm{kHz} \mathrm{Nd:YAG}$ laser (Edgewave IS-400-2-L) and the pulse energy at the exit of the dye laser is $0.6 \mathrm{~mJ}$. A $105 \mathrm{~mm}$-high and $1 \mathrm{~mm}$ thick laser sheet is used to illuminate an axialradial plane through the combustion chamber.

The fluorescence signal from acetone is recorded thanks to a NAC Memrecam HX-3 camera equipped with an image intensifier (Invisible Vision) and a 50 $\mathrm{mm}, \mathrm{f}: 1.8$ lens with a short pass filter $(525 \mathrm{~nm})$. The resulting $768 \times 512$ pixels picture corresponds to one half of the chamber diameter. To record the fluorescence from $\mathrm{OH}$, a Photron Fastcam SA-X2 camera is used with a Lambert HiCATT image intensifier, a $45 \mathrm{~mm}$, f:1.8 UV lens and a 320/40 nm filter. The $1024 \times 1024$ pixels image corresponds to the whole burner diameter. Both cameras record at $10,000 \mathrm{fps}$ with a resolution of $0.135 \mathrm{~mm} / \mathrm{pixel}$ and each camera records 43684 images, corresponding to a little less than 4.4 seconds. This duration corresponds to the size of the buffer memory of the cameras.

A pressure transducer (Kulite WCT-312-5SG) is placed at the dump wall of the combustion chamber and its signal is recorded at $200 \mathrm{kHz}$. Only the part during which the cameras are recording is shown in this study.

Because of the time-resolved nature of the diagnostics, specific post-processing methods are available and are described in the following.

\subsection{Post-processing methods}

Time-resolved data obtained from high-speed $\mathrm{OH}$ and acetone PLIF contain a lot of information but also some noise. In order to extract useful material, namely spatial structures or frequencies in the flame response, Dynamic Mode Decomposition (DMD) is used 
in this study. DMD is a linear analysis technique which extracts monochromatic modes from a set of timeresolved data $[20,21]$. This characteristic makes it very useful for analyzing thermo-acoustic oscillations since they generally offer a narrow frequency response.

DMD modes are complex-valued and depend on both spatial and temporal coordinates. A general form for a DMD mode can be given by the following expression

$$
M(x, t)=K(x) e^{i \phi(x)} e^{(a+i \omega) t}=f_{\text {space }}(x) g_{\text {time }}(t)
$$

where $K(x)$ is a spatially dependent oscillation amplitude, $\phi(x)$ represents a spatially dependent phase and $e^{(a+i \omega) t}$ is the temporal content of the mode, which is a composition of an oscillation and an exponential envelope. In the present studies, the DMD modes that are studied are all steady and thus $a$ is very small, meaning that the temporal part is a simple sinusoidal oscillation $e^{i \omega t}$. It is thus easy to separate the spatial content $f_{\text {space }}$ from the temporal content $g_{\text {time }}$ of the mode.

In the present study, time-resolved diagnostics are used to study a transient operating point. It is thus interesting to obtain instantaneous properties for a given signal and for this purpose, Hilbert transform is used. Given a temporal signal $s(t)$, the Hilbert transform is defined as:

$$
H_{s}(t)=\frac{1}{\pi} \int_{-\infty}^{\infty} \frac{s(\tau)}{t-\tau} d \tau .
$$

The main use of the Hilbert transform lies in the fact that it enables to compute the analytical signal $s_{a}(t)$, defined as:

$$
s_{a}(t)=s(t)+i H_{s}(t) .
$$

$s_{a}(t)$ is a complex signal from which it is possible to extract an instantaneous amplitude $A(t)$ and an instantaneous phase $\phi(t)$ so that $s_{a}(t)=A(t) e^{i \phi(t)}$.

\section{Global behavior of the burner}

As mentioned previously, the studied operating point is transient and corresponds to an increase of the equivalence ratio while maintaining the global flow rate constant. The evolution of the pressure fluctuations in the chamber during this transient operation is shown at the top of Fig. 2. At the beginning of the recording, the peak-to-peak pressure fluctuations are below $300 \mathrm{~Pa}$ while they reach almost $2000 \mathrm{~Pa}$ at the end. In the meantime, the power of the burner has only increased by around $7 \%$, meaning that the increase in pressure fluctuations cannot only be related to the power increase.
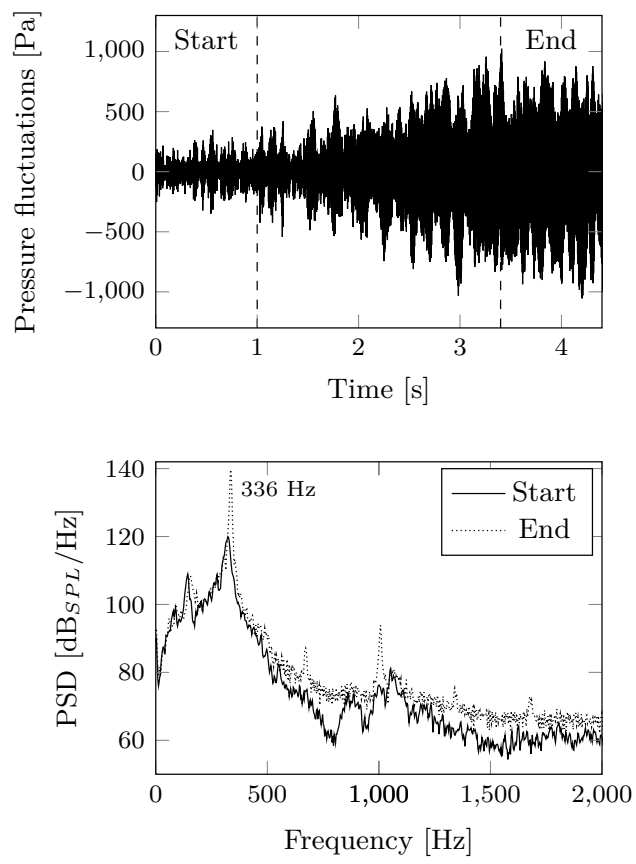

Figure 2: Top: Raw signal of the pressure fluctuations in the combustion chamber during the transient. Bottom: Spectra of the pressure fluctuations signal during the first and the last seconds of the recording.

The bottom of Fig. 2 shows spectra of the pressure signal during the first and last seconds of the recording. These power spectral density estimates are obtained using Welch's method, with 0.33 s long Hamming windows and 50\% overlap. During the first second, the spectrum is characterized by a peak around $324 \mathrm{~Hz}$, indicating moderate thermo-acoustic activity. The spectra for the end of the recording is globally the same as at the start, except for a strong peak at $336 \mathrm{~Hz}$ and its harmonics. This indicates that the strong increase in the pressure fluctuations amplitude is due to coherent fluctuations at this frequency, which corresponds to a strong thermo-acoustic instability. The increase in peak frequency between the start and the end of the recording can be explained by the increase in equivalence ratio which impacts the temperature in the combustion chamber. As the burnt gas temperatures increase, the speed of sound also increases and thus the resonant frequency for the instability follows the same trend.

To monitor major changes in the flame between the beginning and the end of the recording, average images are used. They are shown in Fig. 3 where only the right part of the burner is studied. The left part of the images are the mirror images of the right part of the burner 
averaged during the first second and the right part corresponds to an averaging during the last second.
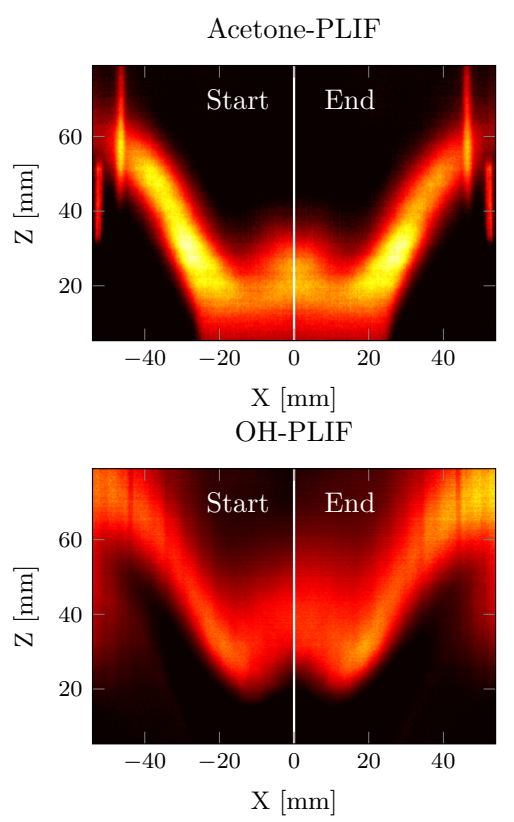

Figure 3: Average images of the right part of the burner taken during the first second (left) and the last second (right) of the recording. The vertical bars in the acetone images are due to laser reflections on the cylindrical chamber.

Whether from acetone-PLIF or OH-PLIF, the average images are similar between the beginning and the end of the recording meaning that no strong change to the flame stabilization occurs because of the change in equivalence ratio or the growth of the thermo-acoustic instability. In the acetone images, the only minor change comes from the top of the arm which shows lower levels than at the beginning of the recording. This tends to indicate that the fresh gases are burnt a bit earlier on average. This is confirmed by the $\mathrm{OH}$ images which show that the intensity at the top of the arm is higher in the end than in the beginning. The $\mathrm{OH}$ images also show higher levels in the burnt gases which is consistent with the equivalence ratio increase.

The dynamics of the PLIF recordings is studied thanks to a Dynamic Mode Decomposition of the data from the end of the recording, where the thermoacoustic instability is the strongest. To combine multiple diagnostics, a stacked-DMD [22] procedure is used where the data from the OH-PLIF, acetone PLIF and pressure transducer are stacked into one matrix that is then processed by DMD. This way, the resulting dynamics come from the information of all diagnostics and the phase reference is the same for all. Bins of $4 \times 4$ pixels are used for the $\mathrm{OH}$ and acetone PLIF images and 10000 snapshots are used, corresponding to the last second of the recording. The DMD mode at $336 \mathrm{~Hz}$ is found to be dominating the others as expected and the parts of it corresponding to the $\mathrm{OH}$ and acetone PLIF are shown in Fig. 4. Since the temporal evolution of the mode corresponds only to a $336 \mathrm{~Hz}$ oscillation, all the interesting information can be found in the spatial parts that are shown in Fig. 4.

For the OH-PLIF image, the full field of view is shown on the left of Fig.4. The dark regions indicate parts of the image where the signal does not fluctuate much with the combustion instability. They correspond to the fresh gases (no signal at all), the burnt gases region and the center part of the flame. This indicates that the thermo-acoustic instability influences almost only the arms of the flame and especially the tip of these arms. Another interesting feature is the symmetry of the image, indicating that two symmetrical parts of the flame fluctuate in phase and with comparable amplitude, as expected for a longitudinal thermo-acoustic instability.

Since the pressure signal is included in the stacked DMD, its phase in the common phase reference given by the DMD can be measured and is around $\frac{\pi}{4}$. This means that the pressure fluctuates in phase with the pink and blue parts of the images. This allows to estimate the Rayleigh index in this case by looking at the OH-PLIF image: the parts of the flame most contributing to the instability are at the top of the arms. This is consistent with other LSB studies reported in the literature [16].

The spatial shape of the acetone part of the stacked DMD mode is shown on the right of Fig. 4. As for the $\mathrm{OH}-\mathrm{PLIF}$ results, some parts of the image are not fluctuating at all. These regions are the burnt gases (no acetone signal), the fresh gases and the center of the flame. The fluctuations mainly happen at the boundaries of the flame arm. An interesting feature is that, for a given position, the phase of the acetone fluctuations is opposite to the one of the $\mathrm{OH}$ fluctuations. This can easily be understood when one takes into account that acetone tracks the fresh gases and $\mathrm{OH}$ the burnt gases. Where there is a surge of fresh gases, there cannot be any burnt gases and conversely. Therefore, the acetone and $\mathrm{OH}$ signals can only have opposite behaviors.

Along the external part of the arm of the acetone image, it is possible to witness a continuous phase decrease in the downstream direction. This phase behavior is typical of the convection of the signal downstream. Indeed, by factoring in a sinusoidal temporal evolution, it is easy to see that such a phase evolution means that the 


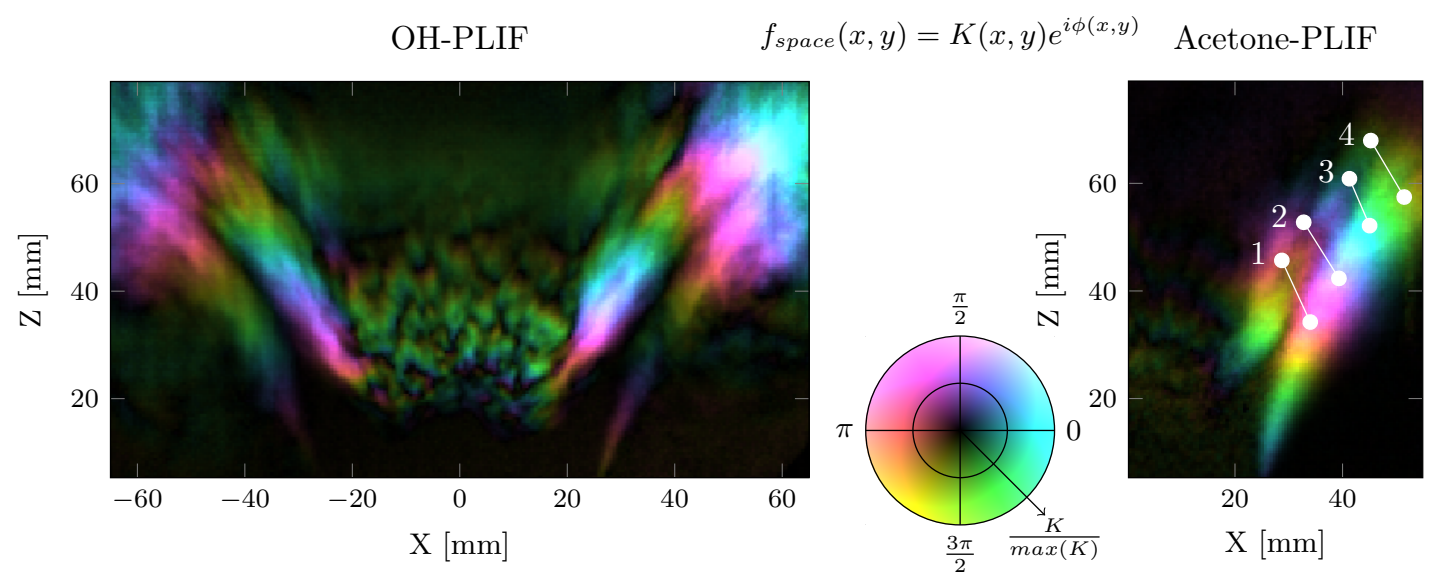

Figure 4: Spatial parts of the $336 \mathrm{~Hz}$ stacked DMD mode corresponding to the OH-PLIF (left) and acetone-PLIF (right) data. They are shown using a colormap that allows to represent both the modulus and angle of the complex quantity. The point couples shown in white are used for Fig. 6.

downstream parts reach a maximum with a delay that increases the further they are from the injector wall. The distance between two green parts in the image, such as (30 mm,20 mm) and (50 mm,55 mm) for example, can be estimated to $40 \mathrm{~mm}$. Since they have the same phase, this means that the convection time between these two parts is one oscillation period at $336 \mathrm{~Hz}$. This gives an estimation of the convection velocity of $13 \mathrm{~m} . \mathrm{s}^{-1}$ which is close to the expected flow velocity at these positions given that the bulk velocity is $10 \mathrm{~m} . \mathrm{s}^{-1}$ and that the velocity in the arms is higher [6]. This result is thus coherent with the findings of Therkelsen et al. [14] that indicate that the instability is linked with the convection of ring vortices from the burner rim.

To sum up, the images in Fig. 4, show that the thermo-acoustic instability is driven by the regular surge of fresh gases at the instability frequency. This excess of reactant is burnt as a burst at the top of the flame, creating a heat release there that is in phase with a higher pressure in the chamber, effectively giving energy to sustain the instability. In that sense, the instability mechanism in the flame arms is similar to what can be found in the literature for laminar premixed flames [23]. This however does not explain why such an instability is triggered when the equivalence ratio is increased. This is the main reason for the analysis carried out in the next section.

\section{Growth of the instability}

Since it was shown that the $\mathrm{OH}$ and acetone-PLIF recordings are quite close, the present section focuses only on the acetone data and thus on the fresh gases dynamics. The acetone-PLIF field of view is divided into
$10 \times 10$ pixels local averaging windows. The values of each of these windows then gives a set of $10 \mathrm{kHz}$ signals that can be studied separately with classical signal processing tools. Each of these signals are divided into 1 -second-long segments and the temporal average is removed so that only the fluctuating part remains. The power spectral density of each segment is estimated using Welch's method with $0.33 \mathrm{~s}$ long Hamming windows with $50 \%$ overlap. The peak frequency of each spectrum is then taken and its value is placed at the spatial position of the averaging window in the images shown in Fig. 5.

For most of the images, the peak frequency is below $200 \mathrm{~Hz}$ indicating that the acetone-PLIF signal is mostly fluctuating at low frequencies. In the beginning of the recording (below 2 seconds), the only part fluctuating around the thermo-acoustic frequency is the outer part of the fresh gases jet. However, closer to the end of the recording (that is, when the thermo-acoustic instability is stronger), it can be seen that the internal part is also fluctuating at $336 \mathrm{~Hz}$. The images in Fig. 5 thus show that the growth of the thermo-acoustic instability is also associated with an evolution of the parts of the flame that react to it: while in the beginning, only the external part is fluctuating, the end shows that the whole arm is fluctuating.

To study the phase relationship between the internal and external parts of the arm, probe points couples are chosen and shown in Fig. 4. Each couple is made of one point in the external part of the arm and one in the internal part. The couples are chosen so that they have the two points have the same phase in the DMD result 

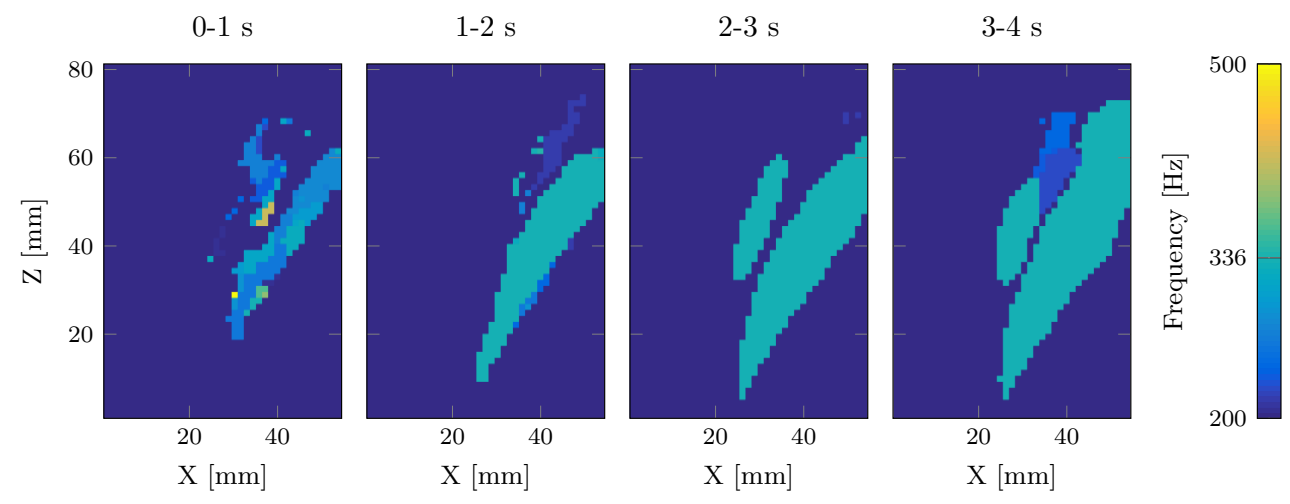

Figure 5: Maps of the peak frequency in the local spectra for four different 1-second-long segments of the acetone-PLIF recordings. The colormap is clipped below $200 \mathrm{~Hz}$ to focus on frequencies around the thermo-acoustic instability.

(ie at the end of the recording) and so that the four couples span one full instability cycle $\left(\frac{\pi}{2}\right.$ separation). The temporal signal from each of these points is bandpass filtered (center frequency: $336 \mathrm{~Hz}$, bandwidth: $30 \mathrm{~Hz}$ ) and its phase is computed by Hilbert transform. The phase difference associated with each couple can thus be obtained. Then, inside 0.1-second-long windows, the probability that the couple is in phase is computed by computing the ratio between the number of samples with a phase difference between $\frac{-\pi}{2}$ and $\frac{\pi}{2}$ and the total number of samples in the window. The evolution of this probability with time is given for the four couples in Fig. 6.

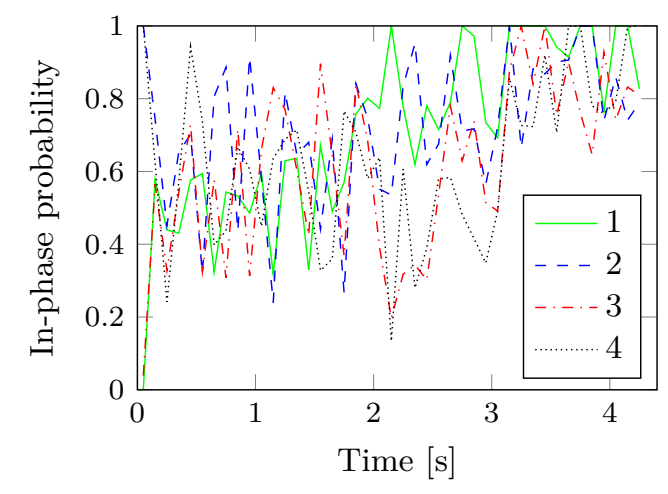

Figure 6: Probability that the point couples shown in Fig. 4 oscillate in phase as a function of time. Point couples from 1 to 4 go from the base of the flame arm to its extremity.

In the beginning, before the internal part of the arm starts to fluctuate at the thermo-acoustic frequency, it can be seen that the probability for all couples is around 0.5 , corresponding to what is expected for two uncorrelated signals. However, as the internal part of the arm starts to react, the in-phase probability of the couples increases until it is almost always above 0.7 at the end of the recording. This indicates that as soon as the internal part of the arm starts to fluctuate at the instability frequency (after 2 seconds, as shown in Fig.5), they are in phase with the external part and thus help to promote the bursts as both fluctuations then arrive at the same time at the top of the arm.

The growth of the instability due to the change in equivalence ratio happens therefore in two steps. First, the ring vortices detaching from the burner rim are acting on the external part of the flame. The timing of the flame burst they create around the top of the external parts of the flame arms is changed because the flame speed is increased by the increase in equivalence ratio which leads to an amplification of the pressure fluctuations. Then, the internal parts of the flame arms start to fluctuate in phase with the external parts and reinforce the bursting mechanism.

\section{Conclusion}

By increasing the equivalence ratio and keeping the bulk velocity in a low-swirl burner, the pressure fluctuations inside the combustion chamber are shown to tremendously increase even though the power change remains limited. This is due to the growth of a thermoacoustic instability at $336 \mathrm{~Hz}$. While this instability does not change the average flame shape or stabilization mechanism, it has a strong impact on the flame arm which starts to periodically release bursts of fresh gases. The subsequent bursts of flame are shown to happen in phase with the high part of the pressure fluctuation cycle, effectively adding energy to the oscillation. Originally generated by the convection of ring vortices de- 
tached from the injector rim, the bursts are then amplified by the fact that the internal and external boundaries of the flame arm fluctuate in phase, which can be related to a change in flame speed.

This mechanism is inferred from the available timeresolved data processed with state of the art methods but would require further studies to be confirmed. For example, since the proposed mechanism is purely kinematic, a turbulent g-equation solver could help in studying it.

\section{Acknowledgments}

We would like to thank Mr Kotaro Moriyama regarding the experimental work on which this study is based. The stay of Antoine Renaud at JAXA and Keio University has been supported by the Erasmus Mundus EASED program (Grant 2012-5538/004-001) coordinated by CentraleSupelec.

\section{References}

[1] A. H. Lefebvre, Journal of Engineering for Gas Turbines and Power 117 (4) (1995) 617-654.

[2] S. Candel, Proceedings of the Combustion Institute 29 (1) (2002) 1-28

[3] C. K. Chan, K. S. Lau, W. K. Chin, R. K. Cheng, Symposium (International) on Combustion 24 (1) (1992) 511-518.

[4] R. K. Cheng, Combustion and Flame 101 (1-2) (1995) 1-14.

[5] W. Nazeer, K. Smith, P. Sheppard, R. Cheng, D. Littlejohn, ASME, 2006, pp. 107-115.

[6] R. K. Cheng, D. Littlejohn, W. A. Nazeer, K. O. Smith, Journal of Engineering for Gas Turbines and Power 130 (2) (2008) 021501.

[7] R. K. Cheng, D. Littlejohn, P. A. Strakey, T. Sidwell, Proceedings of the Combustion Institute 32 (2) (2009) 3001-3009.

[8] D. Littlejohn, R. K. Cheng, D. R. Noble, T. Lieuwen, Journal of Engineering for Gas Turbines and Power 132 (1) (2010) $011502+$.

[9] P. Petersson, J. Olofsson, C. Brackman, H. Seyfried, J. Zetterberg, M. Richter, M. Aldén, M. A. Linne, R. K. Cheng, A. Nauert, Others, Applied optics 46 (19) (2007) 3928-3936.

[10] D. M. Kang, F. E. C. Culick, A. Ratner, Combustion and Flame 151 (3) (2007) 412-425.

[11] S. Tachibana, J. Yamashita, L. Zimmer, K. Suzuki, A. K. Hayashi, Proceedings of the Combustion Institute 32 (2) (2009) 1795-1802.

[12] Y. Huang, A. Ratner, Journal of Propulsion and Power 25 (2) (2009) 365-373.

[13] I. Yilmaz, A. Ratner, M. Ilbas, Y. Huang, International Journal of Hydrogen Energy 35 (1) (2010) 329-336.

[14] P. L. Therkelsen, J. E. Portillo, D. Littlejohn, S. M. Martin, R. K Cheng, Combustion and Flame 160 (2) (2013) 307-321.

[15] M. Emadi, K. Kaufman, M. W. Burkhalter, T. Salameh, T. Gentry, A. Ratner, International Journal of Hydrogen Energy 40 (39) (2015) 13594-13603.

[16] S. Tachibana, K. Kanai, S. Yoshida, K. Suzuki, T. Sato, Proceedings of the Combustion Institute 35 (3) (2015) 3299-3308.
[17] K. Kohse-Höinghaus, R. S. Barlow, M. Aldén, J. Wolfrum, Proceedings of the Combustion Institute 30 (1) (2005) 89-123.

[18] B. Böhm, C. Heeger, R. Gordon, A. Dreizler, Flow, Turbulence and Combustion 86 (3-4) (2011) 313-341.

[19] C. T. Chong, S. Hochgreb, Combustion and Flame 158 (3) (2011) 490-500

[20] P. J. Schmid, Journal of Fluid Mechanics 656 (2010) 5-28.

[21] P. J. Schmid, Experiments in Fluids 50 (4) (2011) 1123-1130.

[22] F. Richecoeur, L. Hakim, A. Renaud, L. Zimmer, Proceedings of the Summer Program (2012) 459.

[23] D. Durox, T. Schuller, S. Candel, Proceedings of the Combustion Institute 30 (2) (2005) 1717-1724. 